\title{
BMJ Open Internet-based, therapist-guided, cognitive-behavioural therapy for body dysmorphic disorder with global eligibility for inclusion: an uncontrolled pilot study
}

Andrew J Gentile, ${ }^{1,2}$ Christopher La Lima, ${ }^{1,2}$ Oskar Flygare, ${ }^{\odot 1,2}$ Jesper Enander, ${ }^{\oplus 1,2}$ Sabine Wilhelm, ${ }^{3}$ David Mataix-Cols, ${ }^{1,2}$ Christian Rück ${ }^{1,2}$

To cite: Gentile AJ, La Lima C, Flygare 0 , et al. Internetbased, therapist-guided, cognitive-behavioural therapy for body dysmorphic disorder with global eligibility for inclusion: an uncontrolled pilot study. BMJ Open 2019;9:e024693. doi:10.1136/ bmjopen-2018-024693

- Prepublication history and additional material for this paper are available online. To view these files, please visit the journal online (http://dx.doi org/10.1136/bmjopen-2018024693).

Received 21 June 2018 Revised 18 October 2018 Accepted 31 December 2018

A) Check for updates

(c) Author(s) (or their employer(s)) 2019. Re-use permitted under CC BY-NC. No commercial re-use. See rights and permissions. Published by BMJ.

${ }^{1}$ Centre for Psychiatry Research, Department of Clinical Neuroscience, Karolinska Institutet, Stockholm, Sweden

${ }^{2}$ Stockholm Health Care Services, Stockholm County Council, Stockholm, Sweden ${ }^{3}$ Department of Psychiatry, Massachusetts General Hospital, Harvard Medical School, Boston, Massachusetts, USA

Correspondence to Dr Christian Rück; christian.ruck@ki.se

\section{ABSTRACT}

Objectives Cognitive-behavioural therapy (CBT) has been shown to be an effective treatment for body dysmorphic disorder (BDD), but access to treatment around the world is limited. One way to increase access is to administer CBT remotely via the internet. This study represents the first effort to remotely deliver a therapist-supported, internet-based CBT treatment with no restrictions on enrolment based on geographical location, and it aims to assess whether this treatment can be delivered safely across international borders, with outcomes comparable to previous BDD-NET trials.

Design Uncontrolled clinical trial.

Participants Patients $(n=32)$ in nine different countries were recruited primarily through internet advertisements. Intervention BDD-NET is a 12-week treatment, consisting of eight treatment modules previously shown to be effective in a Swedish version.

Setting Therapists based at a single, secondary care centre in Sweden provided active guidance and feedback throughout the treatment via asynchronous electronic messages.

Main outcome measure The clinician-administered YaleBrown Obsessive Compulsive Scale for BDD (BDD-YBOCS). Symptom severity was assessed pretreatment, midtreatment (6 weeks), post-treatment and at the 3-month follow-up.

Results There were significant improvements on BDDYBOCS scores $(F(3,71.63)=31.79, p<0.001)$, that were maintained at 3-month follow-up. Mean differences from baseline in BDD-YBOCS scores were -8.12 (week 6), -12.63 (post-treatment) and -11.71 (3-month follow-up). $47 \%$ and $50 \%$ of participants were considered treatment responders at post-treatment and 3-month follow-up, respectively. Additionally, remission rates were $28 \%$ at post-treatment and $44 \%$ at 3-month follow-up. The treatment was also deemed acceptable by patients. Conclusions The results suggest that BDD-NET can be safely and effectively delivered across international borders to a culturally diverse sample. Larger scale randomised controlled trials with more participants from non-Western cultures are warranted to further validate the cross-cultural generalisability of this treatment.

Trial registration number NCT03517384.

\section{Strengths and limitations of this study}

- This is the first study to investigate the feasibility and acceptability of a therapist-guided, internet-based cognitive-behavioural therapy intervention, delivered from a single centre, to an international sample with global eligibility for inclusion.

- The absence of a control condition limits the ability to make inferences about what caused the changes observed.

- Since most participants resided in Western countries, it is unclear to what extent body dysmorphic disorder-NET is generalisable to patients from non-Western cultures.

\section{INTRODUCTION}

Despite the enormous socioeconomic costs and individual suffering caused by mental illness, there are far too few clinicians to meet the global need for mental health services. ${ }^{1}{ }^{2}$ Moreover, outpatient health services are usually open during normal working hours, and this current service model disenfranchises individuals who may have difficulties taking time off work or accessing care if living in remote and underserved areas. Furthermore, issues like stigma, lack of awareness, cost of treatment, and the symptoms of psychiatric disorders themselves can also be barriers to accessing care. ${ }^{3}$ As a result, most individuals with a mental disorder do not receive treatment. ${ }^{4}$

This treatment gap is particularly wide for under-recognised disorders such as body dysmorphic disorder (BDD), where the affected individual is preoccupied with perceived flaws or defects in one's appearance that are not noticeable to others. ${ }^{5}$ In fact, only $10 \%-17 \%$ of those with the disorder report receiving an evidence-based 
psychotherapy like cognitive-behavioural therapy (CBT), despite its common prevalence and significant functional impairment for sufferers. ${ }^{36-10}$

Internet-based CBT (ICBT) aims to increase accessibility and availability to specialised treatment and has been shown to be efficacious and cost-effective for a range of disorders. ${ }^{11}$ While ICBT has been studied for nearly 20 years,${ }^{12}$ there has been an upsurge of promising research on technology-based mental health interventions during the past several years. ${ }^{13}{ }^{14}$ Recently, BDD-NET, a therapist-guided, ICBT programme for BDD, was developed to improve access to evidence-based care, and the treatment has been shown to be safe, efficacious and highly acceptable by patients. ${ }^{1516}$ The treatment is delivered through a secure tailored online platform that contains the treatment content. Communication between therapist and patient is done through asynchronous messaging, requiring only a fraction of therapist time compared with conventional CBT. Crucially, BDD-NET removes key barriers to treatment, while yielding outcomes equivalent to traditional face-to-face CBT. ${ }^{17}$

ICBT represents a promising solution for economically and efficiently targeting mental health disparities around the world. However, this integration of CBT with information technology has yet to realise its true potential to reach underserved populations. Therefore, our aim was to conduct the first investigation evaluating whether a therapist-guided, ICBT intervention could be delivered safely and effectively across international borders, with no geographical restrictions for recruitment. In doing so, the current researchers hope to shed light on aspects of feasibility and ethical considerations that arise in this novel treatment context.

\section{METHODS}

\section{Trial design}

The aim of this investigation was to evaluate the feasibility and safety of a global treatment initiative using an English-language version of BDD-NET. ${ }^{15} 16$ This uncontrolled pilot study was intended to assess different aspects of conducting the study remotely and across international borders; including recruitment, assessment and treatment delivery.

\section{Procedure}

Participants were recruited by clinician referral as well as using internet advertisements through Google AdWords, bddfoundation.org and on internet forums. Individuals interested in participating in the study were directed to the study's website where they provided initial informed consent, and completed an online screening consisting of the Montgomery-Åsberg Depression Rating Scale, Self-Report (MADRS-S), ${ }^{18} 19$ the Body Dysmorphic Disorder Questionnaire (BDDQ), ${ }^{20}$ the Dysmorphic Concerns Questionnaire (DCQ), ${ }^{21}$ the Alcohol Use Disorders Identification Test (AUDIT), ${ }^{22}$ and the Drug Use Disorders Identification Test (DUDIT) ${ }^{23}$ Following this initial screening, eligible individuals were invited for an assessment over VSee, a Health Insurance Portability and Accountability Act (HIPAA) compliant video-conferencing software. During the video-conference assessment, final screening and baseline measures were obtained, as well as verbal informed consent, identification documents and emergency information. Measures administered at this time were the BDD modification of the Yale-Brown Obsessive Compulsive Scale (BDD-YBOCS), ${ }^{24}$ Columbia Suicide Severity Rating Scale (C-SSRS), ${ }^{25}$ Brown Assessment of Beliefs Scale (BABS), ${ }^{26}$ Clinical Global Impressions Scale of Severity (CGI-S) ${ }^{27}$ and Global Assessment of Functioning (GAF). ${ }^{5}$ Additionally, the obsessive-compulsive and related disorders module of the Structured Clinical Interview for DSM- $5^{28}$ and the Mini-International Neuropsychiatric Interview $7^{29}$ were also administered at this time as a means to establish a primary diagnosis of BDD. For full eligibility criteria and details on recruitment and patient flow, see online supplementary appendix A. Eligible participants were then granted access to treatment via the online platform. In order to guarantee participant confidentiality, we used a dedicated server with encrypted traffic and a strong authentication login function.

\section{Participants}

Thirty-two participants were included in the study. These individuals resided in 9 different countries and represented 12 different nationalities (sociodemographic and clinical characteristics of participants are presented in table 1). Inclusion criteria were that participants needed to be aged 18 years or older, meet DSM- 5 criteria for a diagnosis of BDD with symptom severity measuring $\geq 20$ on the BDD-YBOCS, ${ }^{24}$ be outpatient, be fluent in English and have regular access to a computer with an internet connection. Patients who were able to navigate the online registration and screening process were considered to have sufficient computer skills to participate in the study.

Exclusion criteria were concurrent psychological treatment, having received CBT for BDD within 12 months preceding treatment, changes in psychotropic medications within 12 weeks before inclusion, not having access to a 24-hour psychiatric emergency centre in their proximity, or if they could not provide an emergency contact person. Additional grounds for exclusion were current substance dependence, lifetime bipolar disorder or psychosis, MADRS-S score $\geq 35$, personality disorder diagnosis, lifetime history of suicide attempts or clinically significant current suicidal ideation ( $\geq 5$ on item 9 of MADRS-S; C-SSRS (past month)—Most Severe Ideation score $\geq 4$ ). Patients excluded from the study prior to enrolment due to excessive depression or suicidality were subjected to the same safety procedures as patients who were included. They agreed to go to an identified, local 24 hours psychiatric emergency centre in the event that they were at imminent risk and were referred to mental health services in their area for ongoing care. 


Table 1 Sociodemographic and clinical characteristics of
the sample $(\mathrm{N}=32)$

Variable $(\mathrm{N}=32)$

\begin{tabular}{|c|c|}
\hline \multicolumn{2}{|l|}{ Gender, n (\%) } \\
\hline Men & $8(25)$ \\
\hline Women & $24(75)$ \\
\hline Age, mean (SD) & $31.91(7.44)$ \\
\hline \multicolumn{2}{|l|}{ Highest education, $\mathrm{n}(\%)$} \\
\hline Primary school & $1(3.1)$ \\
\hline High school & $6(18.8)$ \\
\hline Bachelor's degree & $14(43.8)$ \\
\hline Master's degree & $10(31.2)$ \\
\hline Doctorate degree & $1(3.1)$ \\
\hline \multicolumn{2}{|l|}{ Occupational status, $\mathrm{n}(\%)$} \\
\hline Working, full time & $9(28.1)$ \\
\hline Working, part time & $10(31.2)$ \\
\hline Student & $7(21.9)$ \\
\hline Unemployed & $5(15.6)$ \\
\hline Disability pension & $1(3.1)$ \\
\hline Years with BDD, mean (SD) & $16.22(9.10)$ \\
\hline No of areas of concern, mean (SD) & $12.16(5.84)$ \\
\hline \multicolumn{2}{|l|}{ Comorbid conditions, $\mathrm{n}(\%)$} \\
\hline Major depressive disorder & $10(31.2)$ \\
\hline Panic disorder & $2(6.2)$ \\
\hline Social anxiety disorder & $5(15.6)$ \\
\hline Generalised anxiety disorder & $5(15.6)$ \\
\hline \multicolumn{2}{|l|}{ Current medication, $\mathrm{n}(\%)$} \\
\hline SSRI & $2(6.2)$ \\
\hline SNRI & $3(9.4)$ \\
\hline Benzodiazepines & $1(3.1)$ \\
\hline Stimulants & $1(3.1)$ \\
\hline Previous psychological treatment, $\mathrm{n}(\%)$ & $25(78.1)$ \\
\hline CBT & $8(32.0)$ \\
\hline PDT & $2(8.0)$ \\
\hline Non-specific counselling & $12(48.0)$ \\
\hline Religious counselling & $1(4.0)$ \\
\hline Unknown & $2(8.0)$ \\
\hline \multicolumn{2}{|l|}{ Plastic surgery } \\
\hline Previous plastic surgery, n (\%) & $13(40.6)$ \\
\hline No of surgeries, mean (SD) & $1.38(2.46)$ \\
\hline \multicolumn{2}{|l|}{ Nationality, n (\%) } \\
\hline American & 12 (37.5) \\
\hline Swedish & $7(21.9)$ \\
\hline Indian & $1(3.1)$ \\
\hline Bulgarian & $1(3.1)$ \\
\hline Finnish & $1(3.1)$ \\
\hline English & $4(12.5)$ \\
\hline
\end{tabular}

Continued

\begin{tabular}{ll} 
Table 1 Continued & \\
\hline Variable & $1(3.1)$ \\
\hline Serbian & $1(3.1)$ \\
South Korean & $1(3.1)$ \\
Irish & $1(3.1)$ \\
Norwegian & $1(3.1)$ \\
Sri Lankan & $1(3.1)$ \\
$\quad$ Lithuanian & $15.63(2.50)$ \\
$\begin{array}{l}\text { Dysmorphic Concerns Questionnaire, } \\
\text { mean (SD) }\end{array}$
\end{tabular}

BDD, body dysmorphic disorder; CBT, cognitivebehavioural therapy; PDT, psychodynamic therapy; SNRI, serotonin and norepinephrine reuptake inhibitor; SSRI, selective serotonin reuptake inhibitor.

\section{Patient and public involvement}

Patients and the general public did not have direct involvement in the design of this study, recruitment or the development of research questions or measures. On publication, patients will be sent a copy of the article which would not have been possible without their participation.

\section{Primary outcome}

The primary outcome was the BDD-YBOCS, administered at baseline, mid-treatment (week 6), post-treatment (week 12) and 3 months after treatment completion. BDD-YBOCS is a semistructured clinician-administered scale, considered to be the gold standard for measuring BDD symptom severity and has demonstrated good psychometric properties. ${ }^{30}$ Scores range from 0 to 48 with higher scores indicating greater severity. Prior to subject enrolment, all evaluators were trained to a reliability criterion (intraclass correlation coefficient of at least. 85) with a gold-standard rater on the BDD-YBOCS.

\section{Secondary outcomes}

Participants with $\geq 30 \%$ reduction on the BDD-YBOCS were considered responders. ${ }^{30}$ Participants no longer meeting full criteria for DSM-5 diagnostic criteria for BDD were considered to be in remission.

Clinicians rated patient overall severity and symptom change on the CGI. The CGI-S ranges from 1 (normal, not ill at all) to 7 (among the most extremely ill of subjects). Similarly, the CGI-I ranges from 1 (very much improved) to 7 (very much worse) ${ }^{27}$ Secondary measures of symptoms included the MADRS-S, ${ }^{18}{ }^{19} \mathrm{GAF}^{5}$ and BABS. ${ }^{26}$ See online supplementary appendix A for a complete list of secondary outcome measures.

\section{Treatment activity, completion and acceptability}

Therapist time spent on the platform reviewing patient progress and responding to messages, the number of messages sent and received, and the number of completed modules were automatically recorded for each patient. Patients rated working alliance every 2 weeks throughout treatment using the Working Alliance Inventory-Short 
Revised (WAI-SR). ${ }^{31}$ At post-treatment, patients rated treatment satisfaction on the client satisfaction inventory (CSI) ${ }^{32}$ Patient credibility and expectancy were also recorded every 2 weeks throughout treatment using the Credibility/Expectancy Questionnaire (CEQ). ${ }^{33} 34$

\section{Adverse events monitoring}

Each week patients were asked if they experienced any adverse events or side effects that could be attributed to treatment (eg, sleep disturbances, increased anxiety or depression symptoms). If so, they were asked to describe them in the form of free text. ${ }^{35}$

For a full list of outcome measures used, as well as a detailed timetable for their administration, see protocol in online supplementary appendix A.

\section{Intervention}

BDD-NET, a 12-week internet-delivered CBT intervention for BDD, was evaluated in Sweden in a pilot study $(n=23)$ and then in a randomised controlled trial $(n=94)$, and showed sustained effects at 2-year follow-up. ${ }^{15} 1636$ It was translated to English for the current study in order to reach an international sample (For a full description of the treatment content, see refs $\left.{ }^{1516}\right)$. Throughout treatment, patients had unlimited access to their therapist from Monday to Friday via asynchronous electronic text messages. The therapist's primary role was to offer clarification and emotional support, and to help participants design and practice exposure and response prevention (EX/RP) exercises that targeted their treatment goals. They also reminded participants to complete treatment content in time via text message reminders. Therapists were doctoral-level psychology students with no previous experience treating BDD, and were supervised by licensed psychologists and psychiatrists based at Karolinska Institutet. Similar to the delivery of the treatment itself, supervision was primarily delivered at least once per week, on a continuous basis, any time that decisions were made related to patient inclusion/exclusion or withdrawal from treatment, any time a patient reported elevated risk, and as needed to address other questions related to the delivery of the treatment itself.

\section{Safety procedures}

Before the start of treatment, researchers verified the 24 hours emergency psychiatric centres in each participant's local area. Symptom levels and adverse events were evaluated weekly via the platform and considered along with patients' message content in order to continuously assess risk. Any increase in suicidal ideation (eg, MADRS-S item $9 \geq 4$ ) was automatically flagged by the system and prompted the therapist for further assessment (see online supplementary appendix A for details on this procedure).

\section{Statistical analyses}

All statistical analyses are reported according to 'intention-to-treat' principles unless otherwise stated. Linear mixed models were used to assess continuous outcomes,

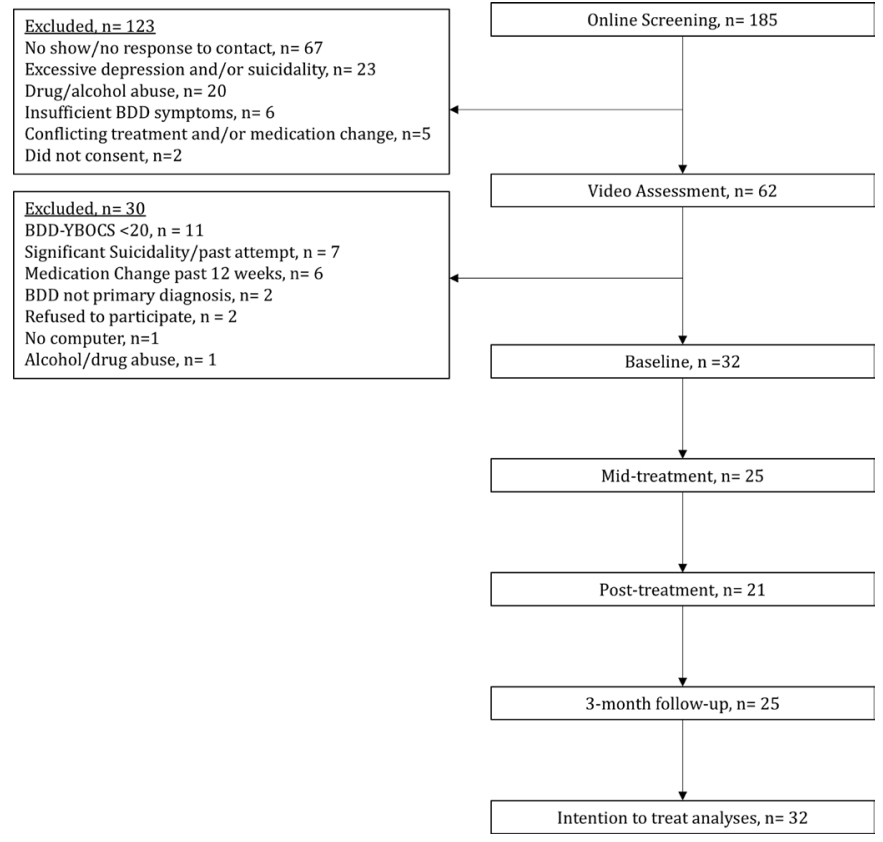

Figure 1 Participant flow through the study. BDDYBOCS, Body Dysmorphic Disorder modification of the YaleBrown Obsessive Compulsive Scale.

with time as a fixed effect and random intercepts for each participant, ${ }^{37}$ and reported using maximum likelihood estimation with 95\% CIs around estimated means. We calculated Cohen's $d$ by dividing the estimated change by the SD of that measure at pretreatment. For non-continuous outcomes, ordinal logistic regression was used with a fixed effect of time, reported as proportional ORs with 95\% CIs. To examine whether data could be deemed to be missing at random, we compared completers (ie, those with BDD-YBOCS data at follow-up) with non-completers on baseline measurements from table 1, using t-tests or $\chi^{2}$ tests where appropriate. Analyses were performed in $\mathrm{R}$ (V.3.4.4) and in SPSS V.25.

\section{RESULTS}

In total, 32 participants initiated treatment, 25 participants $(78 \%)$ completed mid-treatment assessments, $21(66 \%)$ post-treatment and 25 participants $(78 \%)$ follow-up assessments, respectively (see figure 1 for patient flow throughout the study). There were no statistically significant differences between completers and non-completers on baseline demographic and clinical variables $(p=0.29-0.91)$, except that non-completers, on average, had undergone more previous plastic surgeries $(\mathrm{p}=0.03)$.

\section{Primary outcome}

From baseline to week 6, participants made significant improvements on the BDD-YBOCS (estimate -8.12, $95 \%$ CI -10.93 to $-5.32, \mathrm{~d}=1.66, \mathrm{p}<0.001)$. Further improvements were seen at post-treatment (estimate -12.63 , $95 \% \mathrm{CI}-15.61$ to $-9.65, \mathrm{~d}=2.57, \mathrm{p}<0.001)$ and were maintained at the 3-month follow-up (estimate -11.71 , 


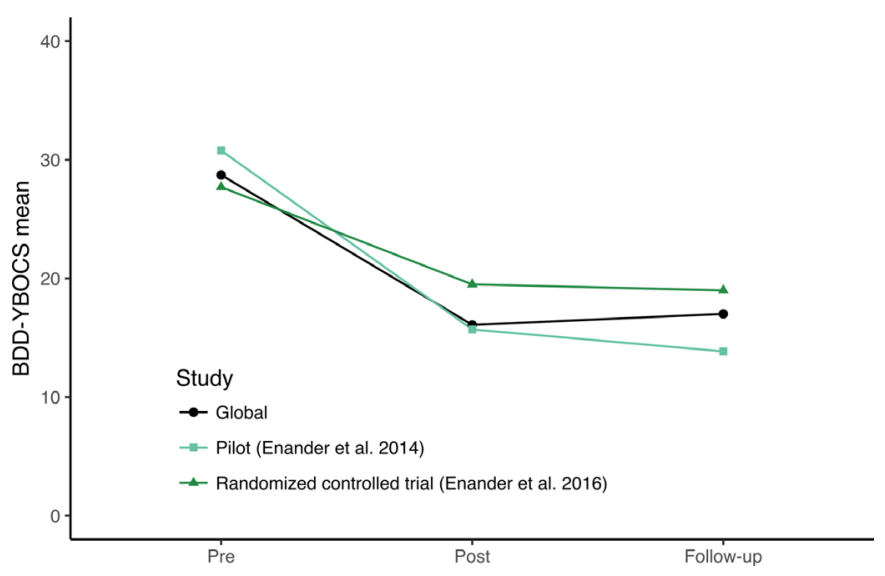

Figure 2 Clinician-rated BDD-YBOCS, comparison with previous BDD-NET trials. BDD-YBOCS, Body Dysmorphic Disorder modification of the Yale-Brown Obsessive Compulsive Scale.

$95 \%$ CI -14.52 to $-8.91, \mathrm{~d}=2.39, \mathrm{p}<0.001)$. The effect of time in a linear mixed effects model was significant $(\mathrm{F}(3$, $71.63)=31.79, \mathrm{p}<0.001)$. These outcomes were similar to those of the previous BDD-NET trials (see figure 2).

\section{Secondary outcomes}

At post-treatment, 15 participants (47\%, 95\% CI $24 \%$ to $70 \%$ ) were considered treatment responders, with $16(50 \%, 95 \%$ CI $29 \%$ to $71 \%)$ participants considered responders at 3-month follow-up. At post-treatment, 9 participants $(28 \%, 95 \%$ CI $7 \%$ to $49 \%)$ no longer met criteria for BDD, which increased to $14(44 \%, 95 \%$ CI $23 \%$ to $65 \%$ ) at the 3-month follow-up.

Participants showed statistically significant improvements on the CGI-S at post-treatment (proportional OR, pOR $0.17,95 \%$ CI 0.06 to $0.47, \mathrm{p}<0.001$ ) and at 3 -month follow-up (pOR $0.22,95 \%$ CI 0.07 to $0.60, p=0.004$ ). The majority of participants who participated in post-treatment and follow-up assessments were much improved or very much improved on the CGI-I after treatment (see figure 3).

Additionally, participants showed significant improvement in depressive symptoms measured using the MADRS-S $(\mathrm{F}(13,243.83)=5.85, \mathrm{p}<0.001)$, global functioning using the $\operatorname{GAF}(\mathrm{F}(2,46.89)=10.46, \mathrm{p}<0.001)$ and

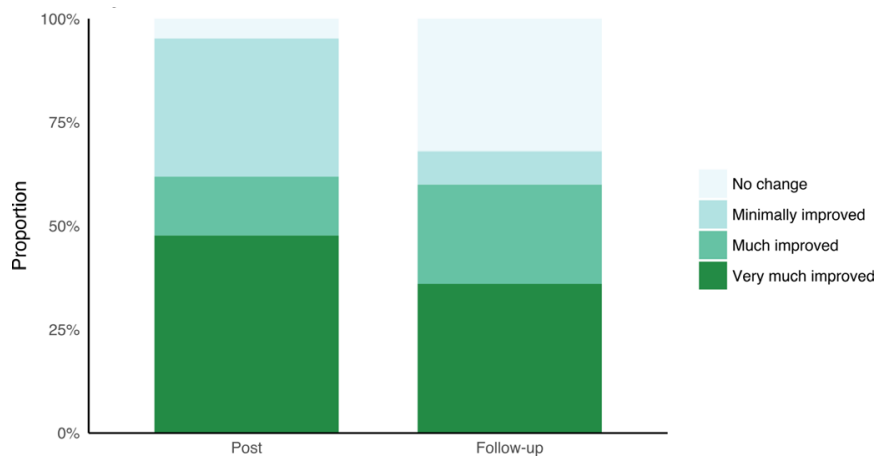

Figure $3 \mathrm{CGl}$ improvement. CGI, Clinical Global Impressions Scale. insight using the BABS $(F(2,47.36)=10.11, p<0.001)$. See table 2 for estimated means and change on primary and secondary outcome measures.

\section{Treatment activity, completion and acceptability}

Therapists spent an average of $15.2 \mathrm{~min}$ supporting patients $(\mathrm{SD}=12.1 \mathrm{~min})$ per participant per week, and sent or received an average of $3.7(\mathrm{SD}=2.7)$ messages per week. For each additional message sent, participants had, on average, a reduction of of 0.11 points on the BDD-YBOCS (95\% CI -0.23 to 0.01 ), but the number of messages sent was not a statistically significant predictor of BDD-YBOCS score when controlling for time $(\mathrm{F}[1,28.80]=3.01$, $\mathrm{p}=0.09)$. In total, $18(56 \%)$ participants completed the core treatment content (modules 1-5). Eight participants $(25 \%)$ completed all eight modules. The mean number of modules completed was 5.1 ( $\mathrm{SD}=2.47)$. Individuals who completed at least five modules had, on average, a lower score on the BDD-YBOCS over time (estimate -6.35, $95 \%$ CI -11.72 to -0.99$)$. The effect of number of modules completed was statistically significant when including time as a covariate $(\mathrm{F}[1,37.62]=5.39, \mathrm{p}=0.03)$. The following results on acceptability measures reflect patient responses at post-treatment which could not be acquired from the entire sample, and therefore, are not intention-to-treat analyses. The mean WAI-SR score after treatment was 49.7 $(\mathrm{SD}=10.7)$ out of a possible 60 , indicating a strong therapeutic bond. Additionally, $95 \%$ of participants who gave feedback at post-treatment $(20 / 21)$ reported that they felt well supported or very well supported by their therapist. Furthermore, despite the fact that some participants were not native English speakers, 95\% of participants found the language used in treatment to be easy or very easy to understand. On average, participants were satisfied with the treatment and found it to be credible. Treatment satisfaction on the CSI was moderate to high at post-treatment, with a mean score of $129.4(\mathrm{SD}=32.6)$ out of a possible 175. Participants rated treatment credibility as moderate on the CEQ at post-treatment (mean=33.1, $\mathrm{SD}=9.8$ ).

\section{Adverse events}

During the course of treatment, (8/32) $25 \%$ of participants reported at least one mild adverse event, which did not pose any acute health risk. This included increased depressive symptoms $(21.9 \%)$, a temporary increase in anxiety $(15.6 \%)$, sleep disturbance or nightmares $(9.4 \%)$ and feelings of shame (6.3\%). Two adverse events needed further action due to increased suicidal ideation. One participant was admitted to high-intensive psychiatric care and ended participation in the study. In this case, researchers facilitated the connection to services in the participant's local area. Another participant who reported a high frequency of suicidal ideation remained in the study and was monitored by a local psychiatrist who had previously treated the patient.

\section{DISCUSSION}

Here, we report the results of the first fully remote, psychological treatment, of BDD or any other disorder, without 
Table 2 Estimated means and change on primary and secondary outcomes

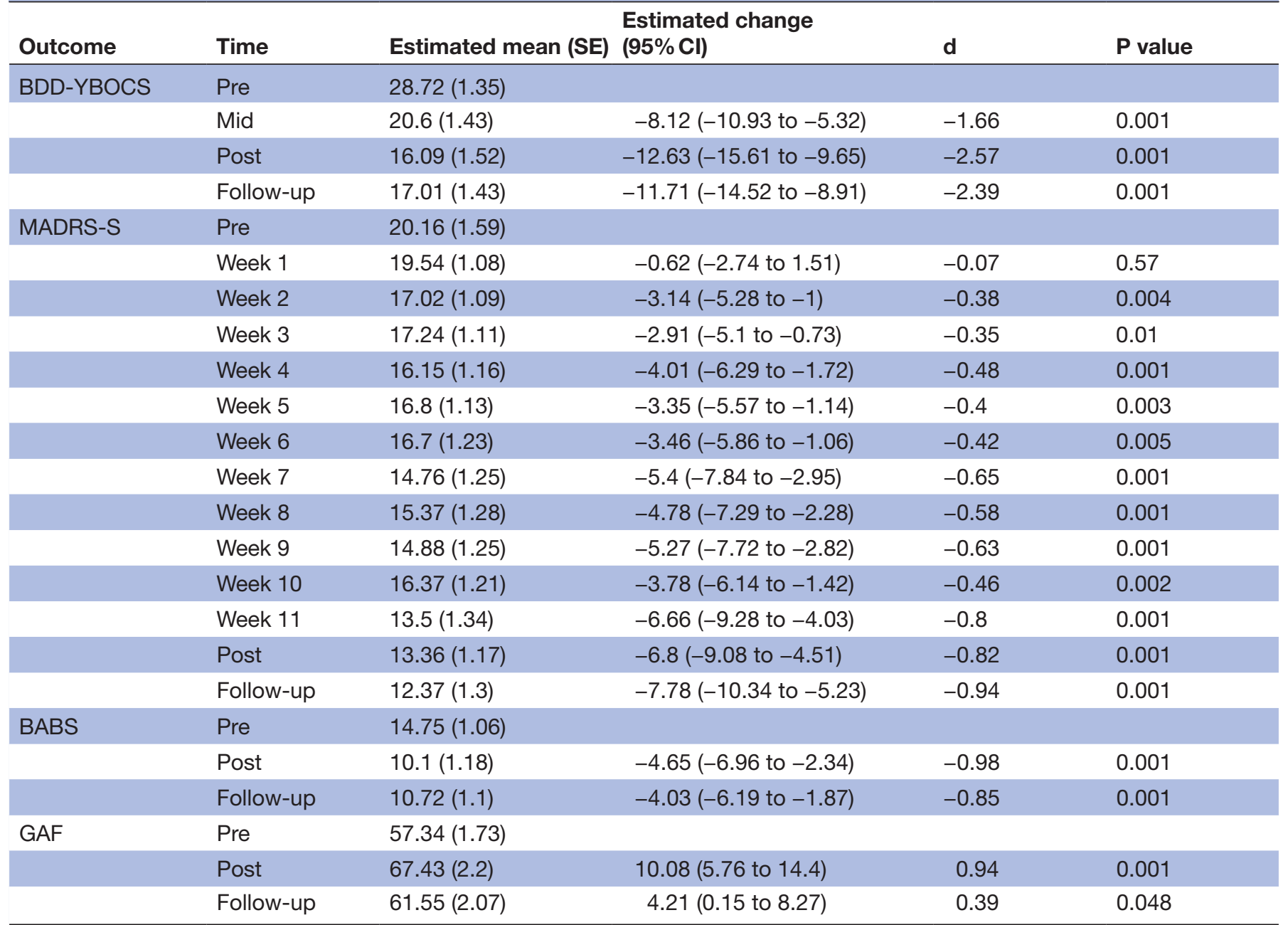

$P$ values: estimated change.

BABS, Brown Assessment of Beliefs Scale; BDD-YBOCS, Body Dysmorphic Disorder modification of the Yale-Brown Obsessive Compulsive Scale; d, Cohen's d; GAF, Global Assessment of Functioning; MADRS-S, Montgomery-Åsberg Depression Rating Scale-Self-Rated.

any geographical restrictions for enrolment. We found that BDD-NET was associated with a large reduction of BDD symptoms at post-treatment and follow-up. Participant-rated reductions in body dysmorphic symptoms and depressive symptoms were $46 \%$ and $34 \%$, respectively. Remission rates were $28 \%$ at post-treatment and $44 \%$ at follow-up. Additionally, patients at post-treatment $(n=21)$ reported a strong therapeutic bond with mean WAI-SR scores at $49.8(\mathrm{SD}=10.4)$ out of a possible 60 . The safety procedures tested in this study worked well. These results indicate that delivering BDD-NET across international borders is feasible, safe and acceptable to clients. Furthermore, as required therapist time was minimal as compared with face-to-face CBT, our findings highlight international ICBT treatment as a promising solution to the global mental health epidemic.

\section{Comparison to previous results}

Current results are in line with previous evaluations of BDD-NET as well as face-to-face CBT for BDD. ${ }^{15-17}$ These findings suggest that delivering BDD-NET across borders in a new language, to a more culturally diverse patient population, had little to no impact on treatment effects. That said, these data are not sufficient to conclude that the treatment effects are universally generalisable. While our sample comprises 12 different nationalities, only $25 \%$ came from non-Western cultures. Post hoc analyses did not identify nationality as a statistically significant predictor of BDD-YBOCS score, but larger samples recruiting more heavily from non-Western countries are needed to detect differences between nationalities and to determine if adaptations should be made to the core treatment content.

\section{Limitations}

While the amount of missing data ( $35 \%$ at post-treatment and $21 \%$ at follow-up) is higher than previous investigations of BDD-NET (4\% at post-treatment and 9\% at follow-up in BDD-NET pilot), it is similar to estimates from recent meta-analyses of both face-to-face and internet CBT. ${ }^{38}$ Furthermore, our sensitivity analysis showed that participants with incomplete data at post-treatment did 
not differ from participants with complete post-treatment data on most baseline measures. However, participants with missing data did report more cosmetic surgeries. This could potentially be related to poorer insight or higher overall severity, which in turn could have impacted their commitment to treatment. Also, since there was no active comparison group, one cannot conclusively say that treatment caused the improvements that were observed. However, this was not the primary aim of the current study since the specific treatment effects of BDD-NET have already been established in comparison with online supportive therapy. ${ }^{16}$

\section{Challenges for clinical trials with global inclusion \\ Legal considerations}

Trials are currently regulated by ethical review boards at universities and healthcare providers. These typically oversee research at their specific site. While multicentre trials may be international, this is to our knowledge the first one-site therapist-guided ICBT treatment study with global eligibility for inclusion. Legislation on ethical vetting is by default national and there are presently no clear guidelines on how trials with international participation of study subjects should be regulated. Internet treatment may also be subject to regulations that govern communications as well as clinical practice. Any legal ambiguity could potentially put some patients at risk when receiving treatment. Therefore, it is essential that international treatment programmes protect patients' privacy and safety in this new context.

\section{Risk management}

Another challenge for studies with global eligibility for inclusion is to ensure adequate care for at-risk patients while also reaching those in need of treatment. While high-risk patients may make clinicians uncomfortable due to liability concerns, many patients seek out ICBT because it is their only viable treatment option. Our procedure for monitoring and responding to suicidality was effective in ensuring patient safety despite the distance between patients and clinicians. One strategy used in this study to manage higher risk patients was to partner with local mental health practitioners who could facilitate risk assessment and expedite a safety plan in their local area if necessary. Psychiatrists can function particularly well in this role, as pharmacological treatment (when indicated) could complement ICBT treatment with minimal redundancy or interference. It is our view that offering remotely delivered evidence-based treatment will always be safer for patients than not having access to treatment at all.

\section{Cultural differences}

International ICBT treatment also poses some novel challenges to cultural competence. Patients not only have different cultural backgrounds, but are currently residing in a different cultural context. Therefore, cultural considerations in treatment may be magnified. Special care should be taken when establishing treatment goals and designing exposure exercises that are culturally appropriate. While our results suggest that a competent therapist can adapt the treatment to the needs of patients from different cultural backgrounds, it should be noted that the participants in this trial were relatively homogeneous (mainly from industrialised nations, highly educated, good command of English language, availability of local psychiatric services). Therefore, it is not yet clear to what extent ICBT can be made available in other settings. Furthermore, while the assessment instruments used in the current study are the most widely used and accepted among BDD researchers, they were developed and validated within Western cultures, with primarily native English speakers. Therefore, it is not yet clear to what extent these instruments assess the same psychological constructs for participants from non-Western backgrounds.

\section{CONCLUSION}

This is, to our knowledge, the first investigation of a fully remote, therapist-guided psychological treatment with recruitment efforts deployed on a global scale. We found large reductions in core BDD symptomatology, with $44 \%$ of patients in remission at follow-up. Participants accepted the treatment and rated their therapist as supportive in the majority of cases. Future trials should evaluate the specific effects of BDD-NET compared with a credible control condition and strive to include more participants from non-Western cultures. In summary, we found that an internet-delivered treatment for BDD can be delivered fully remotely with intact treatment effects, and in a safe way, across countries.

Contributors Author contributions: CR was the primary investigator for the study and drafted the design of the study with CL, JE and DM-C. AJG and CL both independently served as project manager during different periods of time. The treatment manual was written by JE with notable influence from work by SW, and was translated to English by CL. CL also developed the study website, protocol, drafted the ethics submissions and international regulations pertaining to treatment. AJG was in charge of the recruitment, assessment and treatment of participants, with significant contributions by $\mathrm{CL}$ and additional work by $\mathrm{OF}$. Data analysis was primarily conducted by OF and AJG. CR, AJG, CL and OF had full access to data and are guarantors for the accuracy of raw data and statistical analyses. The manuscript was primarily written by AJG, with significant contributions by $O F, C R$, $\mathrm{JE}, \mathrm{SW}, \mathrm{DM}-\mathrm{C}$ and $\mathrm{CL}$.

Funding This study was funded through the regional agreement on medical training and clinical research (ALF) between the Stockholm County Council and Karolinska Institutet. CR was supported by the Swedish Research Council (grant No: K2013-61X-22168-01-3).

Disclaimer The funders had no role in study design; collection, management, analysis and interpretation of data; writing of the report and the decision to submit the report for publication.

Competing interests None declared.

Patient consent for publication Not required.

Ethics approval The central ethical review board in Sweden approved the protocol (CEPN Ö 7-2016), as well as institutional review boards (IRB) at Massachusetts General Hospital (approved 23/112015) and Hofstra University (14/1/2016).

Provenance and peer review Not commissioned; externally peer reviewed.

Data sharing statement № additional data are available. 
Open access This is an open access article distributed in accordance with the Creative Commons Attribution Non Commercial (CC BY-NC 4.0) license, which permits others to distribute, remix, adapt, build upon this work non-commercially, and license their derivative works on different terms, provided the original work is properly cited, appropriate credit is given, any changes made indicated, and the use is non-commercial. See: http://creativecommons.org/licenses/by-nc/4.0/.

\section{REFERENCES}

1. Saraceno B, Saxena S. Mental health resources in the world: results from Project Atlas of the WHO. World Psychiatry 2002;1:40-4.

2. Vigo D, Thornicroft G, Atun R. Estimating the true global burden of mental illness. Lancet Psychiatry 2016;3:171-8.

3. Buhlmann U. Treatment barriers for individuals with body dysmorphic disorder: an internet survey. J Nerv Ment Dis 2011;199:268-71.

4. Kohn R, Saxena S, Levav I, et al. The treatment gap in mental health care. Bull World Health Organ 2004;82:858-66.

5. American Psychiatric Association. Diagnostic and Statistical Manual of Mental Disorders. 5 edn: American Psychiatric Association, 2013.

6. Brohede S, Wingren G, Wijma B, et al. Prevalence of body dysmorphic disorder among Swedish women: a population-based study. Compr Psychiatry 2015;58:108-15.

7. Buhlmann $U$, Glaesmer $\mathrm{H}$, Mewes R, et al. Updates on the prevalence of body dysmorphic disorder: a population-based survey. Psychiatry Res 2010;178:171-5.

8. Rief W, Buhlmann U, Wilhelm S, et al. The prevalence of body dysmorphic disorder: a population-based survey. Psychol Med 2006;36:877.

9. Otto MW, Wilhelm S, Cohen LS, et al. Prevalence of body dysmorphic disorder in a community sample of women. Am J Psychiatry 2001;158:2061-3.

10. Marques L, Weingarden $\mathrm{HM}$, Leblanc $\mathrm{NJ}$, et al. Treatment utilization and barriers to treatment engagement among people with body dysmorphic symptoms. J Psychosom Res 2011;70:286-93.

11. Andersson G, Cuijpers P, Carlbring P, et al. Guided Internet-based vs. face-to-face cognitive behavior therapy for psychiatric and somatic disorders: a systematic review and meta-analysis. World Psychiatry 2014;13:288-95.

12. Andersson G. Using the Internet to provide cognitive behaviour therapy. Behav Res Ther 2009;47:175-80.

13. Thase ME, Wright JH, Eells TD, et al. Improving the efficiency of psychotherapy for depression: computer-assisted versus standard CBT. Am J Psychiatry 2018;175:242-50.

14. Anguera JA, Jordan JT, Castaneda D, et al. Conducting a fully mobile and randomised clinical trial for depression: access, engagement and expense. BMJ Innov 2016;2:14-21.

15. Enander J, Ivanov VZ, Andersson E, et al. Therapist-guided, Internetbased cognitive-behavioural therapy for body dysmorphic disorder (BDD-NET): a feasibility study. BMJ Open 2014;4:e005923.

16. Enander J, Andersson E, Mataix-Cols D, et al. Therapist guided internet based cognitive behavioural therapy for body dysmorphic disorder: single blind randomised controlled trial. $B M J$ 2016;352:i241.

17. Harrison A, Fernández de la Cruz L, Enander J, et al. Cognitivebehavioral therapy for body dysmorphic disorder: a systematic review and meta-analysis of randomized controlled trials. Clin Psychol Rev 2016;48:43-51.

18. Fantino B, Moore N. The self-reported montgomery-Åsberg depression rating scale is a useful evaluative tool in major depressive disorder. BMC Psychiatry 2009;9:26.

19. Svanborg P, Åsberg M. A comparison between the Beck Depression Inventory (BDI) and the self-rating version of the Montgomery Åsberg Depression Rating Scale (MADRS). J Affect Disord 2001;64:203-16.
20. Phillips KA, Atala KD, Pope HGJ. Diagnostic instruments for body dysmorphic disorder. American Psychiatric Association 148th annual meeting, Miami, 1995.

21. Oosthuizen P, Lambert T, Castle DJ. Dysmorphic concern: prevalence and associations with clinical variables. Aust $N Z J$ Psychiatry 1998;32:129-32.

22. Saunders JB, Aasland OG, Babor TF, et al. Development of the alcohol use disorders identification test (AUDIT): who collaborative project on early detection of persons with harmful alcohol consumption--Il. Addiction 1993;88:791-804.

23. Berman $\mathrm{AH}$, Bergman $\mathrm{H}$, Palmstierna $\mathrm{T}$, et al. Evaluation of the Drug Use Disorders Identification Test (DUDIT) in criminal justice and detoxification settings and in a Swedish population sample. Eur Addict Res 2005;11:22-31.

24. Phillips KA, Hollander E, Rasmussen SA, et al. A severity rating scale for body dysmorphic disorder: development, reliability, and validity of a modified version of the Yale-Brown Obsessive Compulsive Scale. Psychopharmacol Bull 1997;33:17-22.

25. Posner K, Brown GK, Stanley B, et al. The Columbia-Suicide Severity Rating Scale: initial validity and internal consistency findings from three multisite studies with adolescents and adults. Am J Psychiatry 2011;168:1266-77

26. Eisen JL, Phillips KA, Baer L, et al. The Brown Assessment of Beliefs Scale: reliability and validity. Am J Psychiatry 1998;155:102-8.

27. Guy W, ECDEU assessment manual for psychopharmacology. Rockville, MD: US Department of Health, Education, and Welfare, Public Health Service, Alcohol, Drug Abuse, and Mental Health Administration, 1976.

28. First MB, Williams JBW, Karg RS, et al. Structured clinical interview for DSM-5 - Research version. Arlington, VA: American Psychiatric Association, 2015.

29. Sheehan DV, Lecrubier Y, Sheehan KH, et al. The Mini-International Neuropsychiatric Interview (M.I.N.I.): the development and validation of a structured diagnostic psychiatric interview for DSM-IV and ICD10. J Clin Psychiatry 1998;59 Suppl 20:22-33.

30. Phillips KA, Hart AS, Menard W. Psychometric evaluation of the yalebrown obsessive-compulsive scale modified for body dysmorphic disorder (BDD-YBOCS). J Obsessive Compuls Relat Disord 2014:3:205-8.

31. Hatcher RL, Gillaspy JA. Development and validation of a revised short version of the working alliance inventory. Psychotherapy Research 2006;16:12-25.

32. Mcmurtry SL, Hudson WW. The client satisfaction inventory: results of an initial validation study. Res Soc Work Pract 2000;10:644-63.

33. Borkovec TD, Nau SD. Credibility of analogue therapy rationales. $J$ Behav Ther Exp Psychiatry 1972;3:257-60.

34. Devilly GJ, Borkovec TD. Psychometric properties of the credibility/expectancy questionnaire. J Behav Ther Exp Psychiatry 2000;31:73-86.

35. Rozental A, Andersson G, Boettcher J, et al. Consensus statement on defining and measuring negative effects of internet interventions. Internet Interv 2014;1:12-19.

36. Enander J, Ljótsson B, Anderhell L, et al. Long-term outcome of therapist-guided internet-based cognitive behavioural therapy for body dysmorphic disorder (BDD-NET): a naturalistic 2-year follow-up after a randomised controlled trial. BMJ Open 2019;9:e024307.

37. Verbeke G. Linear Mixed Models for Longitudinal Data. Linear Mixed Models in Practice. New York, NY: Springer New York, 1997:63-153.

38. Fernandez E, Salem D, Swift JK, et al. Meta-analysis of dropout from cognitive behavioral therapy: magnitude, timing, and moderators. $J$ Consult Clin Psychol 2015;83:1108-22.

39. van Ballegooijen $W$, Cuijpers $P$, van Straten $A$, et al. Adherence to Internet-based and face-to-face cognitive behavioural therapy for depression: a meta-analysis. PLoS One 2014;9:e100674. 